\title{
The Application of Discrete Fourier Transforms in Reducing the Calibration Effort of Indoor Positioning Systems
}

\author{
Anvar Narzullaev $^{1}$, Zahriddin Muminov ${ }^{2}$, Zainidin Eshkuvatov ${ }^{1}$ and Norazmir Mohd Nordin ${ }^{1}$ \\ ${ }^{1}$ Faculty of Science and Technology, Universiti Sains Islam Malaysia \\ ${ }^{2}$ Uzbek-Israel Faculty of High Technology, National University of Uzbekistan
}

\begin{abstract}
For the past decade, Wi-Fi signal fingerprinting algorithm has been considered as a prevalent solution for indoor positioning systems. Fingerprinting based methods require a massive database of Wi-Fi signal samples to calibrate the indoor positioning system and to achieve a high location accuracy. Traditionally the calibration procedure requires human intervention and is very time-consuming, which makes a large-scale deployments of indoor positioning systems non-trivial. Objective of this research to minimise the manual workload by combining the conventional sampling algorithm with signal prediction. In contrast to traditional algorithms, proposed method requires only few signal samples to be collected and rest of the data are approximated using Discrete Fourier Transforms. The main objective of our research is to reduce the calibration effort while maintaining an acceptable location accuracy of the indoor positioning systems.
\end{abstract}

Keywords: indoor positioning systems, wi-fi fingerprinting, RSSI prediction, DFT

\section{INTRODUCTION}

In many scenarios of everyday life and especially warehousing, manufacturing, advertising, navigation and logistics, it is highly desirable to locate and track objects or person quickly and accurately. The market of real-time location systems (RTLS) is growing with tremendous rates and expected to reach US55 billion by 2020 Sakr \& ElSheimy (2017). Currently, the most extensively used and commercially successful location system is the Global Positioning System (GPS), which provides high degree of accuracy and is available worldwide. However, GPS signals originating from satellites suffer from accuracy deterioration and outages in dense urban environments and are almost unavailable indoors (He et al., 2016). The rapid development of $\mathrm{Wi}-\mathrm{Fi}$ wireless local area networks and advent of great mobility have facilitated numerous indoor positioning techniques (Khalajmehrabadi et. al., 2017). Originally designed to provide Internet access to mobile users, 802.11 WLAN standards also emerged as viable alternative for indoor positioning systems.

Indoor environment characteristics and specific requirements of time-based positioning schemes make received signal strength information (RSSI) based positioning techniques more attractive for indoor location estimation (Dwiyasa \& Lim, 2016). The RSSI originated from a wireless access points (AP) is used to estimate the location of a mobile device. All RSSI based localization techniques can be divided into two major categories: signal propagation model and fingerprinting approaches (Salamah et. al., 2016). Since path loss propagation models are prone to large estimation errors due to the random propagation effects in different indoor environments (Narzullaev et. al., 2015), signal fingerprinting approach received most attention from both, academia and industry (Davidson \& Piché, 2017).

Wi-Fi signal fingerprinting is a popular location estimation technology for Wi-Fi based indoor positioning

\footnotetext{
*Corresponding author’s e-mail: zimuminov@gmail.com
} 
systems. The position of mobile users is estimated by matching received signal strength (RSSI) pattern with prerecorded radio map. Although, the fingerprinting is being heavily used by most of the commercially successful indoor positioning systems (Google Indoors, Ekahau, Aeroscout etc.) its main drawback is the time-consuming and exhaustive calibration process. This procedure involves moving around the building and sampling the Wi-Fi signals strength information from nearby wireless access points at pre-defined reference spots. Collected samples stored in a database to build a signal strength map of the region. This procedure carried out manually and it requires tremendous time and efforts to collect signal strength samples for the target area (Jun et. al., 2018).

In this research, we propose a new hybrid approach of constructing RSSI fingerprints database, that combines conventional signal sampling procedure with RSSI estimation using Direct Fourier Transform (DFT). The main objective of this research is to create an accurate and complete Wi-Fi radio signals DB from a limited number of on-site measurements using Discrete Fourier Transform, thus reducing the cost of time-consuming calibration procedure.

The rest of the paper is organized in the following manner. Section II discuss the related work. Section III describes the proposed signal prediction model. The experiment set-up and results are discussed in Section IV. Concluding remarks are given in Section V.

\section{RELATED WORK}

\section{A. Wi-Fi Fingerprinting Based RTLS}

The fingerprint in wireless environment is a feature of the signal, such as RSSI or signal-to-noise ratio (SNR) that varies from one place to another. Combination of such features from multiple radio sources creates a unique pattern of signals specific to a given location. Wi-Fi fingerprinting localization technique is based on comparing the unique signals data from external sources sensed at a particular location with a database of prerecorded data (Davidson \& Piché, 2017).

Wi-Fi fingerprinting works in two stages: offline (calibration) and online (positioning). During the calibration phase the RSSI from surrounding APs at different, predetermined locations are acquired and then stored in a database. This manual procedure is carried out by an operator who has knowledge of the area. Signal DB contains RSSI samples along with MAC address of wireless APs and spot coordinates. In the online positioning phase, the location of mobile device is estimated by comparing the real-time RSSI values from nearby APs, with the values stored in the DB.

An advantage of Wi-Fi fingerprinting is ubiquitous deployment of Wi-Fi access points. Due to rapid growth of wireless networks in indoor environments, Wi-Fi fingerprinting based RTLS does not require any special changes or upgrades to existing network infrastructure (Khalajmehrabadi et. al., 2017). On the other hand, creating a fingerprint DB is often time consuming and, furthermore the newly collected DB may not be reliable if there are major changes to the indoor environment. Consequently, large-scale deployment of fingerprinting RTLS for becomes non-trivial.

\section{B. RSSI Prediction}

Recently, several attempts to reduce the calibration efforts via signal interpolation have been made. These methods generally approximate the missing RSSI data by interpolation of the measurements at neighbouring reference points. In $\mathrm{Hu}$ et. al., (2013) authors presented an efficient database construction method, from relatively sparse measurements, based on low-rank approximation. However, this method starts with assumption that positioning area is represented as a rectangle (to create RSSI measurements matrix) which is not practical for reallife scenarios. Another interesting approach is presented in Lee \& Han (2012). In this research, authors combined signal sampling procedure with Nelder-Mead simplex algorithm. In order to consider signal fading caused by walls and other obstacles, researches refine the approximation model for each cell of target area by a higher-order Voronoi diagram. Although, the proposed method significantly reduces the calibration time, the location accuracy of the model is bit on the downside. Despite the numerous approximation methods mentioned above, there is still potential to improve the existing techniques in terms of increased accuracy and reduced efforts and complexity.

\section{Discrete Fourier Transform}

The discrete Fourier Transform (DFT) converts a spatial 
(real space) description of signal/audio/image data into one in terms of its frequency components called Fourier space description of the data (Broughton \& Bryan, 2018). DFT has been widely deployed by modern video coding standards, for example, MPEG, JVT etc. for multimedia Image/Video/Audio Compression. Although standard DFT technique exist, the focus will be placed on the machine learning regressors.

\section{METHODOLOGY}

\section{A. Reference Signals Sampling}

The proposed Wi-Fi RTLS calibration procedure works in two stages: reference samples collection and RSSI approximation using Direct Fourier Transform. First, we divide the target area into multiple paths, commonly used by people to navigate the building. Next, we allocate multiple reference points along each path, and collect RSSI signal samples originated from surrounding APs. Once we finish the signal sampling, we will create path-specific prediction function using DFT.

\section{B. Direct Fourier Transform}

Let reference points coordinates $\mathrm{x}$ and collected RSSI samples y be vectors defined by

$$
\begin{aligned}
& x^{T}=\left(x_{1}, \ldots, x_{N}\right), \\
& y^{T}=\left(y_{1}, \ldots, y_{N}\right) .
\end{aligned}
$$

where $\mathrm{N}$ is the total number of reference points.

We propose the regression function has a form:

$$
h_{\theta}(x)=\theta_{0}+\sum_{j=1}^{2 m} \theta_{j} c_{j}(x),
$$

where

$$
\theta, \theta^{T}=\left(\theta_{0}, \theta_{1}, \ldots, \theta_{2 m}\right), \quad \text { is }
$$

(unknown)regression parameter vector, $\mathrm{m}$ is a positive integer number and

$$
\begin{aligned}
& c_{0}(x)=1, c_{1}(x)=\cos (x), \ldots, c_{m}(x)=\cos (m x), \\
& c_{m+1}(x)=\sin (x), \ldots c_{2 m}(x)=\sin (2 m x) .
\end{aligned}
$$

It is obvious that the function $h_{\theta}(x)$ in (4) can be describes as a scalar product of two vectors

$$
h_{\theta}(x)=C^{T}(x) \cdot \theta
$$

where $C(x)$ is a vector valued function defined as

$$
C^{T}(x)=\left(c_{0}(x), c_{1}(x), \ldots, c_{2 m}(x)\right) .
$$

The regression parameter vector $\theta$ is defined by the minimum

$$
\min _{\theta} J(\theta)
$$

of the regression cost function

$$
J(\theta)=\frac{1}{2 N} \sum_{k=1}^{N}\left(h_{\theta}\left(x_{k}\right)-y_{k}\right)^{2}
$$

Theorem 1.1. The regression parameter vector $\theta$ has the form

$$
\theta=\left[c c^{T}\right]^{-1} c_{y}
$$

where $C$ is a $N \times(m+1)$ matrix:

$$
c=\left(\begin{array}{cccc}
1 & 1 & \ldots & 1 \\
c_{1}\left(x_{1}\right) & c_{2}\left(x_{2}\right) & \ldots & c_{1}\left(x_{N}\right) \\
\vdots & \vdots & \ddots & \vdots \\
c_{2 m}\left(x_{1}\right) & c_{2 m}\left(x_{2}\right) & \ldots & c_{2 m}\left(x_{N}\right)
\end{array}\right)
$$

Proof. Since $\theta$ is an absolute minimum point of the cost function $J(\theta)$ we consider the system of equations

$$
\begin{gathered}
\frac{\partial J(\theta)}{\partial \theta_{0}}=0, \\
\frac{\partial J(\theta)}{\partial \theta_{1}}=0 \\
\vdots \\
\frac{\partial J(\theta)}{\partial \theta_{2 m}}=0
\end{gathered}
$$

Since

$$
\frac{\partial}{\partial \theta_{0}}\left(h_{\theta}\left(x_{k}\right)-y_{k}\right)^{2}=2\left(h_{\theta}\left(x_{k}\right)-y_{k}\right)
$$

and

$$
\begin{aligned}
& \frac{\partial}{\partial \theta_{j}}\left(h_{\theta}\left(x_{k}\right)-y_{k}\right)^{2}=2 c_{j}\left(x_{k}\right)\left(h_{\theta}\left(x_{k}\right)-y_{k}\right), \\
& j=1, \ldots, 2 m,
\end{aligned}
$$

the equations (9) have the form 


$$
\begin{gathered}
\frac{1}{N} \sum_{k=1}^{N}\left(h_{\theta}\left(x_{k}\right)-y_{k}\right)=0 \\
\frac{1}{N} \sum_{k=1}^{N} c_{1}\left(x_{k}\right)\left(h_{\theta}\left(x_{k}\right)-y_{k}\right)=0, \\
\vdots \\
\frac{1}{N} \sum_{k=1}^{N} c_{2 m}\left(x_{k}\right)\left(h_{\theta}\left(x_{k}\right)-y_{k}\right)=0
\end{gathered}
$$

i.e.

$$
\left(\begin{array}{cccc}
1 & 1 & \ldots & 1 \\
c_{1}\left(x_{1}\right) & c_{2}\left(x_{2}\right) & \ldots & c_{1}\left(x_{N}\right) \\
\vdots & \vdots & \ddots & \vdots \\
c_{2 m}\left(x_{1}\right) & c_{2 m}\left(x_{2}\right) & \ldots & c_{2 m}\left(x_{N}\right)
\end{array}\right)\left[\begin{array}{c}
h_{\theta}\left(x_{1}\right)-y_{1} \\
h_{\theta}\left(x_{2}\right)-y_{2} \\
\vdots \\
h_{\theta}\left(x_{N}\right)-y_{N}
\end{array}\right]=0
$$

Due to the equality in (4) the function $h_{\theta}(x)$ is scalar product of the vector function $C(x)$ and $\theta$.

Consequently, we have

$$
\begin{gathered}
h_{\theta}\left(x_{1}\right)=C^{T}\left(x_{1}\right) \theta, \\
h_{\theta}\left(x_{2}\right)=C^{T}\left(x_{2}\right) \theta, \\
\vdots \\
h_{\theta}\left(x_{N}\right)=C^{T}\left(x_{N}\right) \theta,
\end{gathered}
$$

hence

$$
\left[\begin{array}{c}
h_{\theta}\left(x_{1}\right) \\
h_{\theta}\left(x_{2}\right) \\
\vdots \\
h_{\theta}\left(x_{N}\right)
\end{array}\right]=\left[\begin{array}{c}
C^{T}\left(x_{1}\right) \\
C^{T}\left(x_{1}\right) \\
\vdots \\
C^{T}\left(x_{1}\right)
\end{array}\right] \theta
$$

By virtue of the definition of the matrix $C$, the vector matrix in the last equality satisfy

$$
\left[\begin{array}{c}
C^{T}\left(x_{1}\right) \\
C^{T}\left(x_{1}\right) \\
\vdots \\
C^{T}\left(x_{1}\right)
\end{array}\right]=C^{T}
$$

Therefore,

$$
\left[\begin{array}{c}
h_{\theta}\left(x_{1}\right) \\
h_{\theta}\left(x_{2}\right) \\
\vdots \\
h_{\theta}\left(x_{N}\right)
\end{array}\right]=C^{T} \theta
$$

and hence the equation (11) is reduced to the form

$$
C C^{T} \theta=C_{y}
$$

which provides the equation $\theta$ in (8)
Due to the fact that $\mathrm{J}(\theta)$ is a quadratic form and $\theta$ is a unique critical point, we can claim that $\theta$ is the absolute minimum of the cost function.

\section{RSSI Prediction}

RSSI prediction for any given $\mathrm{x}$ is performed using equations (4), (5) and (8).

\section{RESULTS AND DISCUSSION}

\section{A. Experiment Setup}

The algorithm evaluation is done using Wi-Fi RSSI data samples collected on the 4th floor of the Mines shopping complex located in Serdang, Malaysia. Figure 1 shows the layout of the test area. RSSI samples were collected at 682 reference points, along the five paths with overall length of over 6oom (dash lines). Android devices, with custom software were used for data sampling. The WLAN infrastructure of the building includes more than 250 APs, however only 20 APs with widest coverage and stable signal levels were chosen for further evaluation. In order to have the reliable RSSI DB, we collected 20 samples at each reference points, and the average of 20 measurements is stored in the database. The final DB contains over 13,000 RSSI samples.

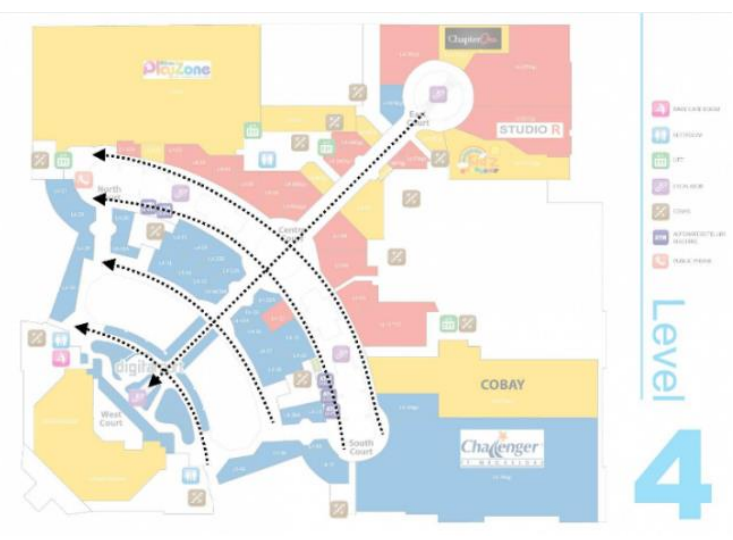

Figure 1. A plan of the building where experiments were conducted.

We compared the proposed method (DFT) with conventional measurement-based algorithm (CONV), as well as, with two recent RSSI prediction-based methods, 
MOSM (Narzullaev \& Park, 2013) and VORO (Lee \& Han, 2012).

\section{B. $\quad$ RSSI Prediction Accuracy}

In the following experiment we have evaluated the proposed model in terms of RSSI prediction accuracy. Since, the main idea behind proposed algorithm is to reduce the calibration time, for further evaluation we gradually decrease the total number of reference points $\left(N_{R P}\right)$. In order to get two times of efficiency $\eta=2$, we reduce the number of RPs from 682 to 341, and we can obtain $\eta=4$ times the efficiency by choosing $N_{R P}=17 O$ and so on.

Figure 2 compares the power spectrum of measured and approximated signals with different number of RPs, for one particular AP, on a single path. Results show that for $\eta=2$ and $\eta=4$ the proposed algorithm can recover the original signal almost identically.
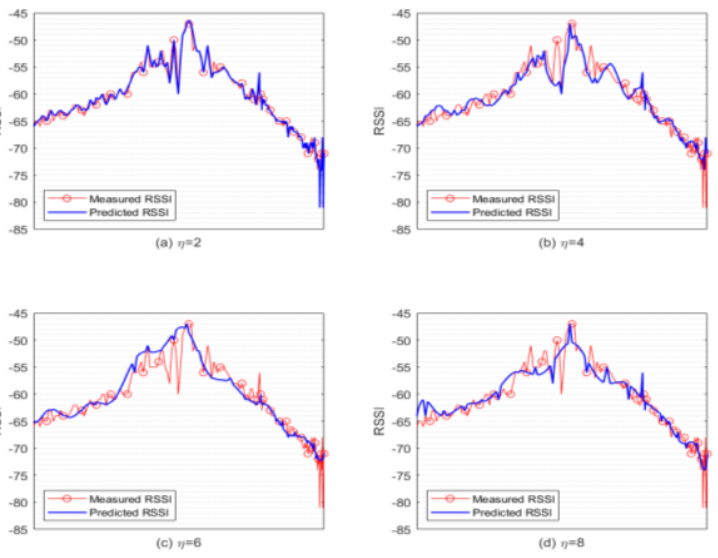

Figure 2. Power spectrum of measured and approximated signals.

Figure 3 shows the cumulative distribution function (CDF) of RSSI prediction error. Results show that even with ten times less measurements $(\eta=10)$, 70\% of the time the proposed algorithm can predict RSSI values with less than $2.5 \mathrm{~dB}$ error.

Next, we compared the proposed algorithm with existing prediction methods. Results in Figure 4 show that the proposed model clearly outperforms the VORO and MOSM, even when $N_{R P}$ is extremely low

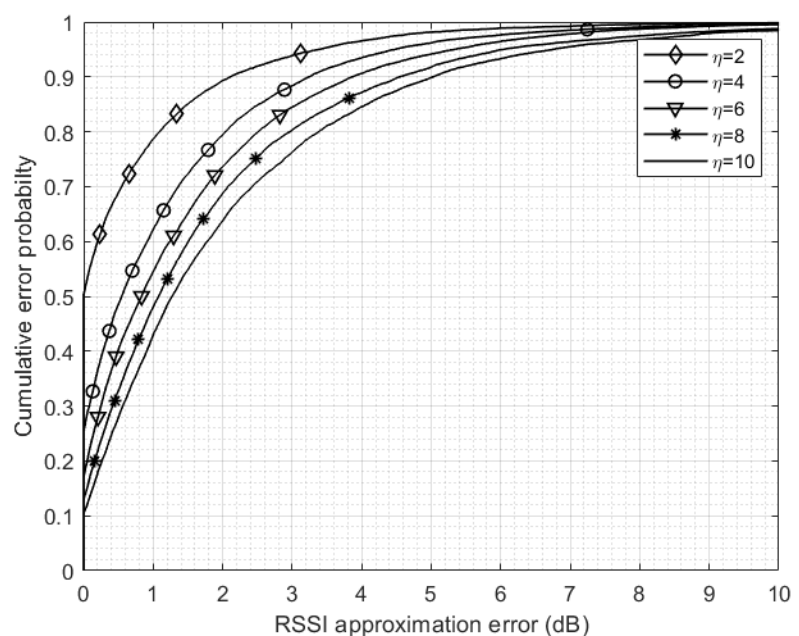

Figure 3. CDF of RSSI prediction accuracy.

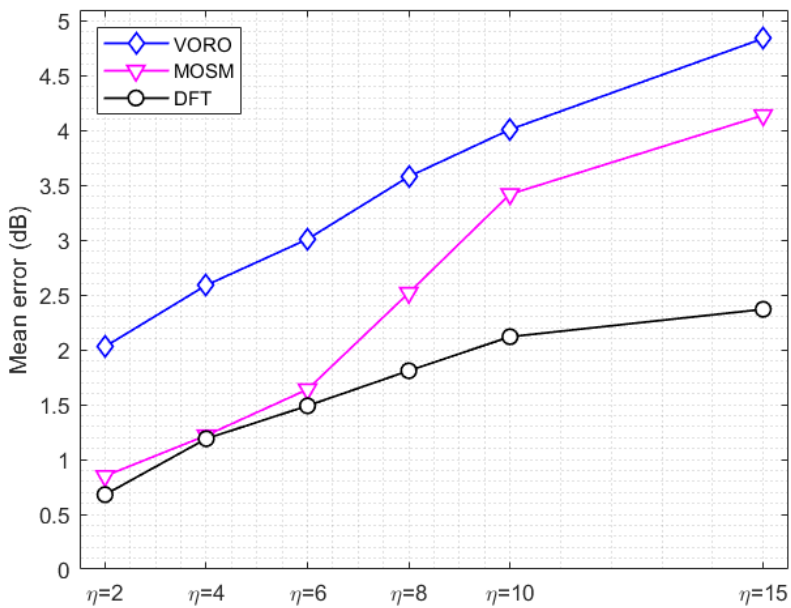

Figure 4. RSSI prediction accuracy of three methods.

\section{Positioning Accuracy}

Next, we evaluate the positioning accuracy or proposed method. We compared the proposed method with conventional (CONV) sampling-based method, as well as, with previously mentioned prediction-based algorithms. Here we adopt the K-nearest neighbour (KNN) algorithm (Narzullaev \& Park, 2013) for location estimation, and we fix the parameter $K=4$. Figure 5 shows the mean location estimation error results. The proposed method (DFT) outperforms the existing algorithms. When $N_{R P}=341$ the mean error was $1.36 \mathrm{~m}$ for DFT, $2.45 \mathrm{~m}$ for MOSM, $2.98 \mathrm{~m}$ for VORO and $2.84 \mathrm{~m}$ for CONV. For the worst-case scenario with $N_{R P}=45$, results were $5.10 \mathrm{~m}$ for DFT, $6.74 \mathrm{~m}$ for MOSM, 10.32m for VORO and $11.20 \mathrm{~m}$ for CONV. 


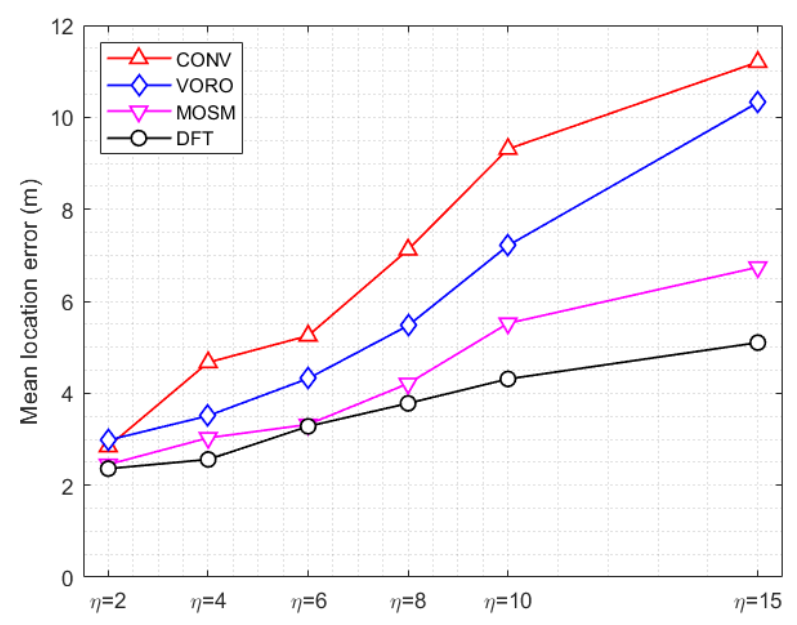

Figure 5. Positioning accuracy results.

\section{CONCLUSION}

In this research we proposed a new Wi-Fi fingerprint DB construction method based on signal approximation using Direct Fourier Transform. The proposed method significantly reduces the calibration effort, compared to conventional, measurement-based algorithm. Real-life field test results showed that proposed method achieves better approximation accuracy than existing predictionbased methods. The constructed fingerprint database was used for positioning and the results showed that the location accuracy is significantly improved compared to conventional, as well as state-of-the-art prediction-based algorithms.

\section{ACKNOWLEDGEMENT}

This work has been supported by USIM research grants PPP/USG-0216/FST/30/17316 and PPPI/FST/0118/051000/15218.

\section{REFERENCES}

Broughton, S. A., Bryan, K. 2018, Discrete fourier analysis and wavelets: Applications to signal and image processing: Second edition. Discrete Fourier Analysis and Wavelets: Applications to Signal and Image Processing: Second Edition.

Davidson, P., Piché, R. 2017, A Survey of Selected Indoor Positioning Methods for Smartphones. IEEE Communications Surveys and Tutorials.

Dwiyasa, F., Lim, M. H. 2016, A survey of problems and approaches in wireless-based indoor positioning. In 2016 International Conference on Indoor Positioning and Indoor Navigation, IPIN 2016.

He, S., Chan, S. H. G. 2016, Wi-Fi fingerprint-based indoor positioning: Recent advances and comparisons. IEEE Communications Surveys and Tutorials.

Hu, Y., Zhou, W., Wen, Z., Sun, Y., Yin, B. 2013, Efficient Radio Map Construction Based on Low-Rank Approximation for Indoor Positioning. Mathematical Problems in Engineering, 2013, 1-9.
Jun, J., He, L., Gu, Y., Jiang, W., Kushwaha, G., Vipin, A., ... Zhu, T. 2018, Low-Overhead WiFi Fingerprinting. IEEE Transactions on Mobile Computing, 17(3), 590-603.

Khalajmehrabadi, A., Gatsis, N., Akopian, D. 2017, Modern WLAN Fingerprinting Indoor Positioning Methods and Deployment Challenges. IEEE Communications Surveys and Tutorials.

Lee, M., Han, D. 2012, Voronoi tessellation-based interpolation method for Wi-Fi radio map construction. IEEE Communications Letters, 16(3), 404-407.

Narzullaev, A., Park, Y. 2013, Novel calibration algorithm for received signal strength based indoor real-time locating systems. AEU - International Journal of Electronics and Communications, 67(7), 637-644.

Narzullaev, A., Nemadaliev, A., Selamat, M. H., Othman, M., Sharif, K. Y. 2015, Wi-fi fingerprint database construction using Chebyshev wavelet functions. In 2015 9th International Conference on IT in Asia, CITA 2015 Proceedings. 
Sakr, M., El-Sheimy, N. 2017, Efficient wi-fi signal strength maps using sparse Gaussian process models. In 2017 International Conference on Indoor Positioning and Indoor Navigation, IPIN 2017.

Salamah, A. H., Tamazin, M., Sharkas, M. A., Khedr, M. 2016, An enhanced WiFi indoor localization System based on machine learning. In 2016 International Conference on Indoor Positioning and Indoor Navigation, IPIN 2016. 